\title{
Satisfação dos usuários com a atenção à saúde bucal em Pauini, Amazonas, Brasil
}

\author{
User's satisfaction with oral care in Pauini, \\ Amazonas, Brazil
}

\author{
Gustavo Sobreira Folhadela' ${ }^{1}$ \\ Lauramaris de Arruda Regis Aranha ${ }^{2}$ (1) \\ Adriana Beatriz Silveira Pinto ${ }^{3}$ (1) \\ Shirley Maria de Araújo Passos 4 (D) \\ Angela Xavier Monteiro 5
}

\begin{abstract}
1-4Universidade do Estado do Amazonas (Manaus). Amazonas, Brasil. gustavosfolhadela@gmail.com, laranha@uea.edu.br, adrianauea@gmail.com, leyshir_br@yahoo.com ${ }^{5}$ Autora para correspondência. Universidade do Estado do Amazonas (Manaus). Amazonas, Brasil. axmonteiro@uea.edu.br
\end{abstract}

RESUMO | OBJETIVO: Este estudo objetivou compreender a satisfação dos usuários com as Equipe de Saúde Bucal das Unidades Básicas de Saúde do município de Pauini, Amazonas. MATERIAL E MÉTODO: Este estudo transversal foi conduzido entre dezembro de 2019 e fevereiro de 2020 nas três unidades básicas de saúde da área urbana do município e teve como participantes os usuários dos serviços de saúde odontológicos. Foram utilizados dois questionários: o primeiro para avaliar o acesso aos serviços de saúde, e outro sobre a satisfação dos usuários com a atenção à saúde bucal. Os resultados foram analisados de modo descritivo por meio de frequências absolutas e relativas. RESULTADOS: Participaram do estudo 265 indivíduos e, ao serem questionados como foi feito agendamento, $61,51 \%$ responderam que se deu por meio de visita à unidade de saúde; com relação ao principal motivo para a última consulta, $47,17 \%$ dos usuários responderam que os principais motivos foram dor de dente e extração. Os percentuais mais elevados de satisfação foram encontrados no domínio estrutura física, e o domínio com menor percentuais foi o de informação e apoio. CONCLUSÃo: os resultados evidenciaram a necessidade de aperfeiçoar a forma de acesso aos serviços de saúde público odontológicos e uma percepção positiva pelos usuários dos serviços de saúde bucal de Pauiní, AM. Entretanto, os melhores resultados foram observados em relação à infraestrutura das unidades de saúde, e foi verificada a necessidade de melhorar o processo de trabalho no que tange às informações e apoio aos usuários dos serviços de saúde bucal.
ABSTRACT | OBJECTIVE: This study aimed to understand user satisfaction with the Oral Health Team of Basic Health Units in the municipality of Pauini, Amazonas. MATERIAL AND METHOD: This cross-sectional study was conducted between December 2019 and February 2020 in the three primary health units in the urban area of the municipality and had as participants the users of dental health services. Two questionnaires were used: the first to assess access to health services and the other about user satisfaction with oral health care. The results were analyzed descriptively utilizing absolute and relative frequencies. RESULTS: Participated in the study 265 individuals, and when asked how scheduling was done, $61.51 \%$ replied that it was through a visit to the health unit, and concerning the main reason for the last consultation, $47.17 \%$ of users said that the main reasons were toothache and extraction. The highest percentages of satisfaction were found in the physical structure domain and the domain with the lowest percentages of information and support. CONCLUSION: The results showed the need to improve the form of access to dental public health services and positive perception by the users of oral health services in Pauiní, AM, however, the best results were observed in the infrastructure of the health units and the need for improvement in terms of information and support for users of oral health services.

KEYWORDS: Unified health system. Community dentistry. Family health strategy.I
PALAVRAS-CHAVE: Estratégia Saúde da Família. Odontologia. Saúde Bucal 


\section{Introdução}

A Estratégia de Saúde da Família (ESF) constitui a estratégia estruturante da atenção básica no Brasil, sendo a porta de entrada preferencial aos serviços de saúde, atuando em equipe multiprofissional e acompanhando a saúde da população adscrita no território de abrangência, incluindo a saúde bucal ${ }^{1}$.

A ESF incorpora e reafirma os princípios básicos do SUS, universalidade de acesso, integralidade na atenção à saúde e participação da comunidade nas decisões 2 . A inclusão da Equipe de Saúde Bucal (ESB) na Estratégia de Saúde da Família se deu no ano 2000 por meio da Portaria número 1.444, a fim de atuar na promoção de saúde, prevenção e controle das diversas doenças que podem acometer a cavidade oral, bem como ampliar o acesso e possibilitar a melhoria nas condições de saúde bucal da população³․

O profissional da equipe de saúde bucal da ESF deve estar apto a atuar em cenários diversos. Além das atividades clínicas do consultório, incluindo visitas e atendimentos domiciliares, orientações familiares, diagnósticos comunitários e planejamento das ações na comunidade, em conjunto com outros membros da equipe, deve também promover educação em saúde. No entanto, o processo de trabalho depende do local, da cultura e da formação curricular, visto que sua participação enquanto profissional ainda é bastante centrada em ações curativas, o que pode dificultar a efetividade de ações de promoção de saúde e do trabalho em equipe multiprofissional, sendo este um dos pilares para a participação do cirurgiãodentista na equipe de saúde da família

Um meio de estimular a manutenção da qualidade e a resolubilidade do sistema de saúde é a realização de avaliações periódicas dos serviços de saúde prestados $^{5}$. A avaliação dos serviços de saúde representa uma ferramenta importante da gestão para aperfeiçoar a qualidade dos serviços executados, e a satisfação do usuário constitui uma parte fundamental destes processos avaliativos, pois permite obter informações da ótica dos usuários sobre a condução dos serviços de saúde ${ }^{6}$. Conhecer a forma como a população adstrita às equipes da ESF avalia o atendimento oferecido contribui para que a equipe possa repensar suas práticas profissionais, visando seu aprimoramento e direcionamento ao atendimento das demandas da população, colaborando para o acesso universal e equitativo. Essa participação do usuário é livre e crítica e pode colaborar para o empoderamento/libertação deste grupo populacional, o que é essencial para viabilizar as políticas de promoção de saúde, prevenção de agravos e controle de enfermidades, além de constituir uma ferramenta de democratização e gestão participativa ${ }^{6,7}$.

Considerando a relevância de avaliar a satisfação dos usuários com os serviços de saúde odontológicos para a melhoria da qualidade e do acesso destes serviços, e a escassez destes estudos em municípios do interior do Estado do Amazonas, este trabalho buscou conhecer a satisfação dos usuários com a atuação das equipes de saúde bucal das unidades básicas de saúde do município de Pauini, interior do estado do Amazonas (AM).

\section{Materiais e métodos}

Este é um estudo transversal de investigação observacional com abordagem quantitativa conduzido no município de Pauini, localizado na mesorregião do Sul Amazonense, região sudoeste do Estado do Amazonas. De acordo com Instituto Brasileiro de Geografia e Estatística (IBGE), a estimativa é de que, em 2020, o município possua uma população de 19.522 habitantes, com um percentual de 51\% residente na área urbana do município. Pauini possui uma área de $41.621 \mathrm{~km}^{2}$ e dista $923 \mathrm{Km}$ da capital, Manaus, em linha reta. Banhado pelo Rio Purus, faz parte da microrregião de Boca do Acre, e tem 64 anos de existência; antes de sua fundação, fazia parte da categoria de Distrito do município de Lábrea ${ }^{\circ}$. Lá, há três Unidades Básicas de Saúde, todas unidades de saúde da Família, que estão localizadas na sede do município, e contam com uma equipe de saúde bucal em cada uma delas, além de uma unidade Hospitalar. A taxa de mortalidade infantil média na cidade é de 22,54 óbitos por mil nascidos vivos. (IBGE, 2017). 
A população de referência do estudo foi composta por usuários do serviço público odontológico, no âmbito da atenção primária à saúde, das três unidades básicas de saúde presentes no município. Os critérios para participação no estudo foram: ter idade igual ou superior a 18 anos, serem usuários das equipes de saúde bucal das unidades básicas de saúde da família, se encontrarem no ambiente da unidade de saúde no momento da coleta, terem mais de uma consulta com a equipe de saúde bucal da unidade e consentirem participar da pesquisa por meio de assinatura do termo de consentimento livre e esclarecido.

O cálculo da amostra foi conduzido utilizando-se o programa Epi info com base na população de 8.616 habitantes do município com a idade supracitada (IBGE); frequência esperada de 50\%, margem de erro de 5\% e efeito de desenho 1,0, nível de confiança de $90 \%$, resultando em um cálculo amostral de 262 indivíduos.

Para condução da pesquisa, foram utilizados dois questionários: o primeiro com dados sociodemográficos dos participantes e acesso aos serviços de saúde, e outro sobre a percepção dos usuários sobre o processo de trabalho das equipes de saúde bucal. O questionário sociodemográfico foi elaborado utilizando questões sociodemográficos dos usuários e de acesso aos serviços de saúde odontológica, extraídas da Pesquisa Nacional de Saúde (PNS, 2013). Para avaliação da percepção dos usuários acerca do processo de trabalho das equipes de saúde bucal, foi utilizado um questionário estruturado (Bordin et al., 2017), contendo doze (12) questões relacionadas ao objeto do estudo, agrupadas em quatro domínios, sendo: duas questões referentes ao cuidado médico, três questões referentes à informação e apoio, quatro questões sobre organização dos serviços, e três questões relativas a estrutura física. As questões apresentavam os padrões de resposta: satisfeito, mais ou menos satisfeito e insatisfeito ${ }^{10}$.

A coleta de dados foi conduzida nos meses de dezembro de 2019 e janeiro e fevereiro de 2020, através de entrevistas de forma individual, em dias e turnos alternados (manhã e tarde, em local neutro no interior das unidades de saúde, ou externo às suas dependências, a fim de evitar constrangimento por parte dos entrevistados pela proximidade com os profissionais do serviço de saúde odontológicos. No total, 265 indivíduos participaram do estudo.

Os dados coletados foram estratificados e analisados por meio de planilhas Excel (Microsoft Office 2010). A análise estatística foi realizada de modo descritivo por meio de frequências absolutas e relativas.

Este estudo foi aprovado pelo Comitê de Ética em Pesquisa com Seres Humanos da Universidade do Estado do Amazonas-UEA, sob número CAAE 13198819.0.0000.5016, respeitando os ditames do Conselho Nacional de Saúde Brasileiro e normas internacionais para pesquisas com seres humanos. Todos os participantes foram informados sobre os objetivos do estudo, métodos de coleta, sobre sua voluntariedade, assim como do sigilo, e aquiesceram em participar da pesquisa por meio da assinatura do termo de consentimento livre e esclarecido.

\section{Resultado}

Participaram do estudo 265 indivíduos com idade entre 18 e 74 anos, obtendo-se média de idade de 33,78 anos, sendo $47(17,74 \%)$ do sexo masculino, e 218 $(82,26 \%)$ do sexo feminino; $232(87,55 \%)$ residiam na área urbana e 33 (12,46\%) na área rural do município (tabela 1).

Com relação ao principal motivo para a última consulta odontológica, para 67,93\% (180) dos usuários, o principal motivo era dor de dente e extração; em apenas $16,60 \%$ (44) dos pacientes, o motivo era raspagem e alisamento radicular, revisão ou manutenção, e 13,96\% (37) buscavam por tratamento. Ao serem questionados sobre a forma como conseguiram a consulta, 62,26\% (165) agendaram previamente, e $29,43 \%$ (78) foram direto ao serviço de saúde (demanda espontânea). No que tange ao modo como foi feito o agendamento, $25,66 \%$ (68) relataram que foi agendado na consulta anterior, e 61,51\% (163) por meio de visita à unidade de saúde, conforme podemos observar na Tabela 1. 
Tabela 1. Perfil sociodemográfico e acesso aos serviços de saúde bucal pelos usuários do município de Pauini-AM, 2020

\begin{tabular}{|c|c|c|}
\hline Variáveis & $\mathbf{n}$ & $\%$ \\
\hline \multicolumn{3}{|l|}{ Gênero } \\
\hline Masculino & 47 & 17,74 \\
\hline Feminino & 218 & 82,26 \\
\hline \multicolumn{3}{|l|}{ Moradia } \\
\hline Urbana & 232 & 87,55 \\
\hline Rural & 33 & 12,45 \\
\hline \multicolumn{3}{|l|}{ Motivo da última consulta odontológica } \\
\hline Dor de dente, extração & 180 & 67,93 \\
\hline Tratamento & 37 & 13,96 \\
\hline Limpeza, revisão, manutenção & 44 & 16,60 \\
\hline Prótese & 3 & 1,13 \\
\hline Fazer radiografia & 1 & 0,38 \\
\hline \multicolumn{3}{|l|}{ Como conseguiu a consulta odontológica } \\
\hline Direto ao serviço de saúde & 78 & 29,43 \\
\hline Agendou previamente & 165 & 62,26 \\
\hline Encaminhado pela unidade de saúde & 14 & 5,28 \\
\hline Encaminhando por outro serviço & 4 & 1,51 \\
\hline Outros & 4 & 1,51 \\
\hline \multicolumn{3}{|l|}{ Agendamento } \\
\hline Deixou agendado da consulta anterior & 68 & 25,66 \\
\hline Visita a Unidade para marcar consulta & 163 & 61,51 \\
\hline Por telefone & 2 & 0,75 \\
\hline $\begin{array}{l}\text { Outros (urgência/ACS/terceiros/encaixe/dia do } \\
\text { funcionário) }\end{array}$ & 32 & 12,08 \\
\hline Total & 265 & 100,00 \\
\hline
\end{tabular}

A seguir, na tabela 2, evidencia-se a satisfação dos usuários com a atenção à saúde bucal. No domínio do cuidado médico, os maiores percentuais de satisfação se concentraram no atendimento pelo dentista e equipe (83,02\%); na organização dos serviços, os melhores índices se concentraram na satisfação com o horário que o dentista trabalha; entretanto, os maiores percentuais de insatisfação foram evidenciados no tempo em que o paciente fica aguardando na sala de espera antes de ser chamado (26,79\%). A estrutura física foi o domínio com melhores resultados, e a informação e apoio tiverem os menores percentuais; sendo a questão das instruções do dentista para prevenir as principais doenças da boca a que apresentou menores resultados de satisfação neste domínio (76,60\%). 
Tabela 2. Satisfação dos usuários com a atenção à saúde bucal, do município de Pauini, AM, 2020

\begin{tabular}{|c|c|c|c|}
\hline Domínios & Satisfação & $\mathrm{n}$ & $\%$ \\
\hline \multicolumn{4}{|c|}{ Cuidado Médico } \\
\hline \multirow{2}{*}{$\begin{array}{l}\text { Atendimento pelo } \\
\text { dentista e equipe } \\
\text { nessa unidade }\end{array}$} & Satisfeitos & 220 & 83,02 \\
\hline & $\begin{array}{l}\text { Não satisfeitos/ } \\
\text { mais ou menos }\end{array}$ & 45 & 16,98 \\
\hline \multirow{2}{*}{$\begin{array}{l}\text { Resolução dos } \\
\text { problemas de saúde } \\
\text { bucal }\end{array}$} & Satisfeitos & 208 & 78,49 \\
\hline & $\begin{array}{l}\text { Não satisfeitos/ } \\
\text { mais ou menos }\end{array}$ & 57 & 21,51 \\
\hline \multicolumn{4}{|c|}{ Organização dos serviços } \\
\hline \multirow[b]{2}{*}{$\begin{array}{l}\text { Horário de trabalho } \\
\text { do cirurgião-dentista }\end{array}$} & Satisfeitos & 232 & 87,55 \\
\hline & $\begin{array}{l}\text { Não satisfeitos/ } \\
\text { mais ou menos }\end{array}$ & 33 & 12,45 \\
\hline \multirow{2}{*}{$\begin{array}{l}\text { Tempo de espera para } \\
\text { agendar a sua } \\
\text { consulta }\end{array}$} & Satisfeitos & 192 & 72,45 \\
\hline & $\begin{array}{l}\text { Não satisfeitos/ } \\
\text { mais ou menos }\end{array}$ & 64 & 24,15 \\
\hline \multirow{3}{*}{$\begin{array}{c}\text { Consegue marcar a } \\
\text { consulta com o } \\
\text { dentista }\end{array}$} & Satisfeitos & 219 & 82,64 \\
\hline & $\begin{array}{l}\text { Não satisfeitos/ } \\
\text { mais ou menos }\end{array}$ & 46 & 17,36 \\
\hline & Satisfeitos & 194 & 73,21 \\
\hline $\begin{array}{c}\text { Tempo que aguarda } \\
\text { na sala de espera }\end{array}$ & $\begin{array}{l}\text { Não satisfeitos/ } \\
\text { mais ou menos }\end{array}$ & 71 & 26,79 \\
\hline \multicolumn{4}{|c|}{ Estrutura física } \\
\hline \multirow{2}{*}{$\begin{array}{c}\text { Limpeza dos } \\
\text { ambientes em que o } \\
\text { dentista e sua equipe } \\
\text { o atendem }\end{array}$} & Satisfeitos & 264 & 99,62 \\
\hline & $\begin{array}{l}\text { Não satisfeitos/ } \\
\text { mais ou menos }\end{array}$ & 1 & 0,38 \\
\hline \multirow{2}{*}{$\begin{array}{c}\text { Conforto dos } \\
\text { ambientes em que o } \\
\text { dentista e sua equipe } \\
\text { o atendem }\end{array}$} & Satisfeitos & 233 & 87,92 \\
\hline & $\begin{array}{l}\text { Não satisfeitos/ } \\
\text { mais ou menos }\end{array}$ & 32 & 12,08 \\
\hline \multirow{2}{*}{$\begin{array}{c}\text { Sinalização do } \\
\text { consultório do } \\
\text { dentista, facilidade de } \\
\text { localização }\end{array}$} & Satisfeitos & 253 & 95,47 \\
\hline & $\begin{array}{l}\text { Não satisfeitos/ } \\
\text { mais ou menos }\end{array}$ & 12 & 4,53 \\
\hline \multicolumn{4}{|c|}{ Informação e apoio } \\
\hline \multirow{2}{*}{$\begin{array}{l}\text { Esclarecimento de } \\
\text { dúvidas durante o seu } \\
\text { atendimento } \\
\text { odontológico }\end{array}$} & Satisfeitos & 204 & 76,98 \\
\hline & $\begin{array}{l}\text { Não satisfeitos/ } \\
\text { mais ou menos }\end{array}$ & 61 & 23,02 \\
\hline $\begin{array}{c}\text { Informações sobre } \\
\text { prevencão das }\end{array}$ & Satisfeitos & 203 & 76,60 \\
\hline $\begin{array}{l}\text { principais doenças da } \\
\text { boca }\end{array}$ & $\begin{array}{l}\text { Não satisfeitos/ } \\
\text { mais ou menos }\end{array}$ & 62 & 23,40 \\
\hline \multirow{2}{*}{$\begin{array}{l}\text { Esclarecimento de } \\
\text { dúvidas após o } \\
\text { tratamento com o } \\
\text { dentista }\end{array}$} & Satisfeitos & 219 & 82,64 \\
\hline & $\begin{array}{l}\text { Não satisfeitos/ } \\
\text { mais ou menos }\end{array}$ & 46 & 17,36 \\
\hline Total & & 265 & 100,00 \\
\hline
\end{tabular}




\section{Discussão}

Este trabalho se propôs a avaliar a satisfação dos usuários dos serviços públicos de saúde bucal do município de Pauini, interior do Amazonas. O processo de avaliação da qualidade em serviços de saúde envolve a participação de diferentes atores que fazem parte da produção de cuidado em saúde bucal, sendo o usuário um ator que tem papel crucial neste processo. O olhar sob o prisma do usuário em relação ao cuidado em saúde bucal traz à luz questões relevantes, muitas vezes não percebidas pelos profissionais. Assim, torna-se essencial verificar como estes atores percebem a qualidade dos serviços que estão sendo prestados, visando verificar e analisar os diferentes pontos de vista e, posteriormente, buscar planejar e organizar os serviços para alinhá-los de modo a atender às necessidades de ambos (usuários e profissionais) e obter a melhoria de forma permanente.

Em relação ao perfil dos participantes do estudo, a grande maioria foi do sexo feminino e residente na área urbana da cidade, concordando com os estudos de Santos et al., 2015ㅌ, Moimaz et al., 20151ㅡ, e Brunhauser et al., 2013눈. Uma possível explicação para este fato pode ser atribuída ao fato de as muIheres buscarem a atenção à saúde com maior frequência que os homens.

Quanto ao acesso aos serviços de saúde odontológicos, a maioria dos usuários conseguiu a consulta agendando previamente; entretanto, uma parcela se dirigiu diretamente ao serviço de saúde, logo, o agendamento da consulta foi feito por meio de visita à unidade em mais da metade dos casos. Estes resultados podem refletir a necessidade de se aprimorar a organização da demanda na atenção à saúde bucal de forma a contribuir com melhor acesso e, consequentemente, resolutividade nos atendimentos em saúde bucal na atenção básica do município, por ser considerada a porta de entrada preferencial da população ao serviço de saúde ${ }^{12}$. Além disso, pode-se constatar o baixo percentual de agendamentos pelos agentes comunitários de saúde, dada a relevância do papel deste ator junto à equipe multidisciplinar de saúde da família, principalmente no âmbito das visitas domiciliares, sendo que uma possível estratégia para melhorar este panorama se concentra na educação permanente como ferramenta para maior integração entre equipe de saúde bucal e agentes comunitários de saúde.
Quanto ao principal motivo da consulta, foi relatado um elevado percentual de busca por atendimento por dor de dente e extração. Estes resultados podem refletir a necessidade de as equipes atuarem com enfoque na atenção integral à saúde bucal dos usuários, aprimorando e ampliando ações de promoção da saúde, educação e prevenção, simultaneamente à resolução das necessidades de procedimentos curativos da comunidade, para minimizar a atenção à saúde bucal mutiladora, que acaba por colaborar para a deterioração da saúde bucal ao longo do tempo.

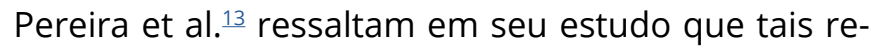
sultados podem estar relacionados à cultura da sociedade ou até mesmo da saúde ofertada, onde há procedimentos de mutilação, sem ou com mínima adesão das novas diretrizes da municipalização e integralização dispostas pelo SUS. Neste contexto, o estudo de Roselino et al. ${ }^{14}$ propôs que a falta de conhecimento por parte da comunidade adstrita aos serviços de ESF, bem como da Saúde Bucal, faz com que se tenha uma limitação por busca de tratamentos paliativos e conservadores, tendo em vista o expressivo número de exodontias na cidade pesquisada no trabalho de Roselino, onde muitos relataram que procuram a UBS somente quando sentem dor (odontalgias) ou têm o dente quebrado (fraturado), resultado semelhante ao presente estudo.

A satisfação é definida através do ato ou expressão de satisfazer ou ser satisfeito, explicado, coerente, de contentamento. Assim, cada usuário, sendo ele o foco principal dos programas e políticas de saúde, através de sua avaliação nas políticas públicas, pode fiscalizar o sistema público de saúde ${ }^{10}$. Dessa forma, a participação da população em busca dos direitos é legal e necessária, e a comunidade deve estar sempre atenta aos seus problemas de saúde e aos dos serviços, reivindicando soluções para um melhor funcionamento.

A satisfação dos usuários pode refletir o modo de trabalho das equipes de saúde bucal, bem como a participação e envolvimento da gestão local. Neste estudo, encontrou-se elevado percentual de satisfação nos quatro domínios analisados. Entretanto, com percentuais mais elevados no domínio estrutura física e resultados inferiores no domínio informação e apoio, o qual se refere ao esclarecimento de dúvidas e informações transmitidas aos usuários sobre prevenção de doenças bucais, e tratamentos realizados. 
Desta maneira, além dos atendimentos curativos, ressalta-se a necessidade da atuação do profissional na prevenção de doenças bucais, por meio de educação e promoção de saúde ${ }^{15}$, o que pode impactar na integralidade do cuidado.

Salientado os dados supracitados, Bordin et al., 201710, em seu estudo, destacam achados semeIhantes, sendo positivos quanto ao nível de satisfação dos usuários participantes; além disso, sua avaliação foi direcionada, além dos usuários, aos profissionais: Cirurgião Dentista (CD), Técnico de Saúde Bucal (TSB) e Agentes Comunitários de Saúde (ACS), sendo encontrados menores valores de satisfação por parte dos ACSs, o que reflete na capacidade crítica e visão ampliada aos serviços de saúde de modo geral.

Neste estudo, todas as unidades de saúde do município são unidades de saúde da família, o que pelas características de seu processo de trabalho, como atuação com uma população adscrita, acompanhamento longitudinal, criação de vínculo com a comunidade, pode ter contribuído com os resultados positivos encontrados, o que vem de encontro ao estudo de Brunhauser et al.12, o qual conduziu pesquisa comparativa da satisfação dos usuários da ESF com os usuários da UBS, e houve uma maior porcentagem de satisfação daqueles pacientes assistidos pela Estratégia de Saúde da Família. A avaliação da prestação de serviços na estratégia da família será sempre valiosa para resolução de possíveis problemas no sistema de saúde municipal em toda a federação brasileira, principalmente para minimizar a desigualdade na busca de saúde como prevenção de agravos físicos e psi-


no domínio informação e apoio ressaltam a necessidade de aperfeiçoamento no processo de trabalho relacionado à educação em saúde e informações aos usuários pelos profissionais da equipe de saúde bucal, a fim de contribuir para o autocuidado bem como para o empoderamento individual e comunitário e diminuir as necessidades de tratamento curativo em detrimento do preventivo.

Ainda, a satisfação positiva dos usuários obtida neste estudo pode estar relacionada também com a baixa condição socioeconômica do município, o que torna as pessoas menos críticas, porém mais cooperativas em relação ao meio na sociedade inserida ${ }^{16,17}$. A relação da baixa escolaridade dos participantes também é citada em outros artigos e, neste assunto, uma das explicações para tal, mediante as construções teóricas referenciadas, pode ser pelo nível de informação ou desconhecimento sobre seus direitos garantidos, onde muitos pensam estar tendo uma doação ou ajuda, mas o Sistema de Saúde Brasileiro garante o acesso e continuidade dos serviços ofertados de forma gratuita, universal e integral, desde a atenção primária subsequentemente aos demais níveis de atenção ${ }^{18}$.

Outras possíveis limitações em relação aos resultados deste estudo podem se referir ao constrangimento dos participantes em responder às questões relativas ao serviço de saúde, por estarem no local, mesmo com garantia de sigilo e anonimato. Ademais, pode-se citar a subjetividade de algumas questões, o que pode contribuir para dificuldade de compreensão por parte dos participantes.

A despeito de tais limitações, este estudo contribui com avaliação do acesso aos serviços de saúde odontológicos, assim como a percepção dos usuários sobre a atenção à saúde bucal ofertada no município de Pauini, o que pode colaborar para o aprimoramento da qualidade dos serviços ofertados, haja vista a lacuna de estudos referente à temática em questão nos municípios do interior do Estado do Amazonas.

Além disto, em uma pesquisa realizada no município de Panamirim-RN19 (2010), sobre os desafios do Cirurgião-Dentista na Estratégia Saúde da Família, pode-se refletir que este modelo possibilitou melhor atenção à saúde bucal, havendo melhora na organização do serviço, humanização, assistência e prevenção das doenças bucais e acesso, sendo que tais características, resultantes do modelo de atenção à saúde, no presente estudo, também puderam contribuir para a elevada satisfação encontrada.

Estudos citados na descrição deste trabalho trazem à luz a percepção do usuário sobre os serviços de saúde, e estes conceitos são fundamentais para a organização da atenção à saúde bucal, com vista à atenção integral para a saúde das pessoas e de toda a comunidade, incentivando a participação da sociedade no planejamento das ações em saúde coletiva ${ }^{20,21}$; ressalta-se a importância dessa avaliação ocorrer de forma permanente. Além desse processo, é fundamental haver trabalho contínuo de educação e divulgação de políticas públicas de saúde bucal, disseminando o conhecimento sobre os seus direitos e deveres nas comunidades do município de Pauini, AM. 
Na pesquisa de Fadel et al. 22 , um dos fatores para a satisfação dos usuários da saúde pública odontológica relatado no seu estudo foi de caráter social e econômico, no qual, por meio de questionamentos, comparou-se os relatos dos profissionais com os investimentos no custeio de materiais por parte do Governo Municipal, Estadual e Federal. Comparando esse contexto com a atual pesquisa em Pauini, Amazonas, um município de pequeno porte com economia baseada na subsistência familiar, e com uma Prefeitura Municipal com baixas verbas de tributos/ impostos locais coletados e dependência maior dos repasses financeiros Estaduais e Federais, podendo gerar um funcionamento do sistema de saúde local com limitações de materiais, estrutura e equipamentos. Portanto, todo o investimento na área de Saúde pode refletir na satisfação dos munícipes e na atuação dos profissionais de forma produtiva, começando da gestão, de forma humanitária e positiva, pois é uma necessidade da Saúde em todo o território brasileiro, principalmente na prevenção de doenças e educação em saúde por meio da execução de políticas públicas.

Só há mudanças no modelo assistencial se houver mudanças na produção do cuidado pelos profissionais, para que ele passe a enxergar o usuário como um ser único, integral, e sem colocá-lo dentro de normas que aprisionam o desenvolvimento deste processo da avaliação de satisfação 11,23 . Deste modo, cada profissional da saúde bucal na atenção básica do município deve assumir o papel transformador das práticas de saúde vigentes, em práticas que privilegiem o cuidado e a humanização, com uma visão ampliada do processo saúde-doença, a execução dos programas de saúde e atuação autêntica e integral, conforme dispõem a Portaria do Ministério da Saúde $n^{\circ} 2979$ de 2019, que estabelece novo modelo de financiamento de custeio da Atenção Primária à Saúde no âmbito do Sistema Único de Saúde ${ }^{24}$.

\section{Conclusão}

Os resultados deste estudo evidenciaram uma percepção positiva dos usuários quanto aos serviços de saúde bucal de Pauini, AM, apesar de seu pequeno porte populacional e de sua localização geográfica distante de grandes centros urbanos. É importante ressaltar os resultados positivos em relação à infraestrutura das unidades de saúde e à necessidade de melhora no que se refere à informação e apoio, evidenciando a necessidade de aperfeiçoar o processo de comunicação entre equipe de saúde, usuários e ações de educação em saúde bucal.

No que se refere ao acesso aos serviços de saúde odontológicos, os resultados ressaltaram necessidade de aperfeiçoar a organização do acesso aos usuários, objetivando contribuir com o processo de trabalho e acolhimento pelas equipes de saúde bucal. Assim, recomenda-se uma avaliação de forma contínua, incluindo a percepção dos usuários em relação aos serviços de saúde, de forma a aprimorar a participação destes atores da avalição dos serviços de saúde, e colaborar para o aprimoramento dos processos de trabalho e consequentemente uma atenção à saúde bucal integral e de qualidade à população do município.

\section{Contribuições dos autores}

Folhadela GS desempenhou a elaboração da pesquisa, coleta e análise dos dados escrita dos resultados e discussão. Monteiro AX participou da análise dos dados e redação dos resultados e discussão. Aranha LAR, Pinto ABS, Passos SMA atuaram na escrita dos resultados e discussão.

\section{Conflitos de interesses}

Nenhum conflito financeiro, legal ou político envolvendo terceiros (governo, empresas e fundações privadas, etc.) foi declarado para nenhum aspecto do trabalho submetido (incluindo, mas não se limitando a subvenções e financiamentos, participação em conselho consultivo, desenho de estudo, preparação de manuscrito, análise estatística, etc.).

\section{Referências}

1. Ministério da Saúde (Brasil). Memórias da Saúde da Família no Brasil. 1a ed. Brasília: Ministério da Saúde; 2010.

2. Ministério da Saúde (Brasil). Sistema Único de Saúde (SUS): estrutura, princípios e como funciona. Brasília: Ministério da Saúde; 2019;2(3.0)

3. Reis WG, Scherer MDA, Carceri DL. O trabalho do CirurgiãoDentista na Atenção Primária à Saúde: entre o prescrito e o real. Saúde debate. 2015;39(104):56-64. https://doi.org/10.1590/0103110420151040608 
4. Mattos GCM, Ferreira EF, Leite ICG, Greco RM. A inclusão da equipe de saúde bucal na Estratégia Saúde da Família: entraves, avanços e desafios. Ciênc. Saúde Coletiva. 2014;19(2):373-82. https://doi.org/10.1590/1413-81232014192.21652012

5. Souza TMS, Roncalli AG. Saúde bucal no Programa Saúde da família: uma avaliação do modelo assistencial Saúde bucal no Programa Saúde da Família: uma avaliação do modelo assistencial. Cad. Saúde Pública. 2007;23(11):2727-39. https://doi. org/10.1590/S0102-311X2007001100020

6. Santos MLMF, Cruz SS, Gomes-Filho IS, Soares JSP, Figueiredo ACMG, Coelho CM. Satisfação dos usuários adultos com a atenção em saúde bucal na estratégia de saúde da família. Cad. Saúde Coletiva. 2015;23(2):163-71. http://dx.doi.org/10.1590/1414$\underline{462 \times 201500020057}$

7. Morosini MVGC, Fonseca AF, Lima LD. Política Nacional de Atenção Básica 2017: retrocessos e riscos para o Sistema Único de Saúde. Saúde debate. 2018;42(116):11-24. http://dx.doi. org/10.1590/0103-1104201811601

8. Instituto Brasileiro de Geografia e Estatística (Brasil). IBGE Cidades [Internet] [citado em 2019 mai 12]. Disponível em: https://cidades.ibge.gov.br/brasil/am/pauini/panorama

9. Instituto Brasileiro de Geografia e Estatística (Brasil). Diretoria de Pesquisas [Internet]. Coordenação de Trabalho e Rendimento. Gerência de Pesquisa Anual. Pesquisa Nacional de saúde 2013 [citado em 2019 abr 10]. Disponível em: https://www.pns.icict. fiocruz.br/arquivos/Novos/Questionario\%20PNS.pdf

10. Bordin D, Fadel CB, Moimaz SAS, Garbin CAS, Saliba NA. Estudo comparativo da satisfação de usuários e profissionais da saúde com o serviço público odontológico. Ciênc. Saúde Coletiva. 2017;22(1):151-60. http://dx.doi.org/10.1590/1413$\underline{81232017221.13522015}$

11. Moimaz SAS, Rós DT, Bordin D, Rovida TAS, Garbin CAS. Satisfação e perfil de usuários do serviço odontológico no Sistema Único de Saúde. RFO. 2015;20(3):334-39. http://dx.doi. org/10.5335/rfo.v20i3.5466

12. Brunhauser AL, Magro ML, Neves M. Avaliação de serviços de saúde bucal: um estudo comparativo. RFO. 2013;18(1):24-3. https://doi.org/10.5335/rfo.v18i1.2850

13. Pereira DQ, Pereira JCM, Assis MMA. A prática odontológica em Unidades Básicas de Saúde em Feira de Santana (BA) no processo de municipalização da saúde: individual, curativa, autônoma e tecnicista. Ciênc. Saúde Coletiva. 2003;8(2):599-609. http://dx.doi.org/10.1590/S1413-81232003000200020

14. Roselino PL, Damasceno JL, Figueiredo GLA. Saúde bucal na atenção primária à saúde: articulações entre o ensino e a estratégia de saúde da família. Rev Odontol UNESP. 2019; 48:e20190081. https://doi.org/10.1590/1807-2577.08119
15. Farias MR, Sampaio JJC. Papel do cirurgião-dentista na equipe de saúde da família (Internet). Rev Gaucha Odontol. 2011;59(1):109-15. Disponível em: http://www.revistargo.com.br/ include/getdoc.php?id=6063\&article=996

16. Aquilante AG, Aciole GG. O cuidado em saúde bucal após a Política Nacional de Saúde Bucal - "Brasil Sorridente": um estudo de caso. Ciênc. Saúde Coletiva. 2015;20(1):239-48. http://doi. org/10.1590/1413-81232014201.21192013

17. Moimaz SAS, Marques JAM, Saliba O, Garbin CAS, Zina LG, Saliba NA. Satisfação e percepção do usuário do SUS sobre o serviço público de saúde. Physis Revista de Saúde Coletiva. 2010;20(4):1419-40. https://doi.org/10.1590/141381232014201.21192013

18. Cerdeira LCR, Groismann S. Qualidade dos serviços de saúde bucal sob a perspectiva do usuário. Rev. bras. Odontol. [Internet]. 2014;71(2):203-07. Disponível em: http://revodonto.bvsalud.org/ $\mathrm{pdf} / \mathrm{rbo} / \mathrm{v} 71 \mathrm{n} 2 / \mathrm{a} 19 \mathrm{v} 71 \mathrm{n} 2 . \mathrm{pdf}$

19. Costa RC, Medeiros-Júnior A, Costa ICC, Pinheiro IVA. Processo de Trabalho do Dentista na Estratégia de Saúde da Família do Município de Parnamirim-RN: Enfrentando os Desafios de um Novo Modelo de Atenção.Rev Odontol Bras Central [Internet]. 2010;19(51):327-32. Disponível em: http://files.bvs.br/ upload/S/0104-7914/2011/v19n51/a2536.pdf

20. Moimaz SAS, Burili MC, Bordin D, Garbin CAS, Saliba TA, Saliba NA. Satisfação dos usuários segundo variáveis de organização dos serviços públicos odontológicos. Arch Health Invest. 2017;6(1):149. https://doi.org/10.21270/archi.v6i1.1776

21. Protasio APL, Gomes LB, Machado LS, Valença AMG. Satisfação do usuário da Atenção Básica em Saúde por regiões do Brasil: $1^{\circ}$ ciclo de avaliação externa do PMAQ-AB. Ciênc. Saúde Coletiva. 2017;22(6):1829-44. https://doi.org/10.1590/1413$\underline{81232017226.26472015}$

22. Fadel CB, Bordin D, Santos CB, Carvalho DR, Moimaz SAS. Users' satisfaction with the public dental service: the discovery of new patterns. Cad. Saúde Coletiva. 2019;27(2): 172-18. https://doi. org/10.1590/1414-462×201900020008

23. Santos NML, Hugo FN. Formação em Saúde da Família e sua associação com processos de trabalho das Equipes de Saúde Bucal da Atenção Básica. Ciênc. Saúde Coletiva. 2018;23(12):431929. https://doi.org/10.1590/1413-812320182312.12922016

24. Ministério da Saúde (Brasil). Portaria n².979, de 12 de novembro de 2019. Diário Oficial da União. Ed. 220. Brasília: Ministério da Saúde. 2019; Seção 1. 97 p. 